\title{
Analysis on Psychosocial Disorder in Children During Post-COVID Education - A Machine Learning Approach
}

\author{
Dillip Narayan Sahu1, Pankajini Sahu ${ }^{2 *}$ \\ ${ }^{1}$ Lecturer, Department of MCA, Gangadhar Meher University (GMU), India \\ ${ }^{2}$ Lecturer, Department of Education, ShreeRam College, Rampur, Sonepur, Odisha, India
}

\begin{abstract}
As SARS COVID is continues to spread in the world also in India as well, the growth rate of detection of COVID cases might be non-exponential but the actual cases and data and its effect towards the new generation especially in the case of children, the impact is a very serious issue. The current situation not only affects the economic and health condition of the country but also in the psychological context to the whole society across the nation. In the situation of a complete lockdown, a kind of restriction to the children for there open movements may be restricted from playing, schooling, any kind of physical contact with outsiders, socialization are all directly or indirectly leading to a more isolated behavioral impact towards the brain or mindset of the children. In this paper, we have shown some real-time experiments and observations with the help of some predictive analysis and algorithms which clearly shows the impact of COVID in children as a real case of Psychosocial Disorder.
\end{abstract}

Keywords: Algorithm, COVID, Education, Machine Learning, Psychosocial Disorder.

\section{INTRODUCTION}

Child Psychology- This defines how the period of the growth of people changes in time as day by day from the birth through adolescence and also why this changes occurs. 1 year old kid, 2 year old baby, 3 year old boy, 4 year old girl child, 5 year old boy child, if we take a close look on all these cases then it will very much clear to be confine that the mental social psychosocial physical cognitive emotional developments are differ person to person with respect to changes in time[1][2].

From the statistical data given by National Mental Health Programme (NMHP), about 6 to 7\% of the population are suffering from mental disorders. If we believe on the report of the World Bank 1993, the DALY- Disability Adjusted Life Year loss due to the neural disorder is much more greater than other diseases combinedly such as - malaria, worm infestations, diarrhea and tuberculosis[3][4].

The following events are of the greatest significance with respect to children and their families in a pandemic[5].

1. Death or physical injury to a family member.

2. A loss of home or possessions.

3. Relocation (school changes).

4. Job loss.

5. Parental disorganization or dysfunction.

Factors Affecting Responses

1. Perceived or actual life threat.

2. Duration of life disruption.

3. Familial and personal property loss.

4. Parental reactions and extent of family disruption.

5. Child's predisaster state.

6. Probability of recurrence.

\section{EXPERIMENTS AND OBSERVATIONS}

We have taken a dataset name=Child.arff (Attribute Relation File Format) as our input and generate different results based on different machine learning algorithms.

2.1 NAME=Weka.filters.supervised.attribute.Discretize SYNOPSIS 
An instance filter that discretizes a range of numeric attributes in the dataset into nominal attributes. Discretization is by Fayyad \& Irani's MDL method (the default)[6].

CAPABILITIES

Class -- Binary class, Nominal class

Attributes -- Binary attributes, Date attributes, Empty nominal attributes, Missing values, Nominal attributes, Numeric attributes, Relational attributes, String attributes, Unary attributes

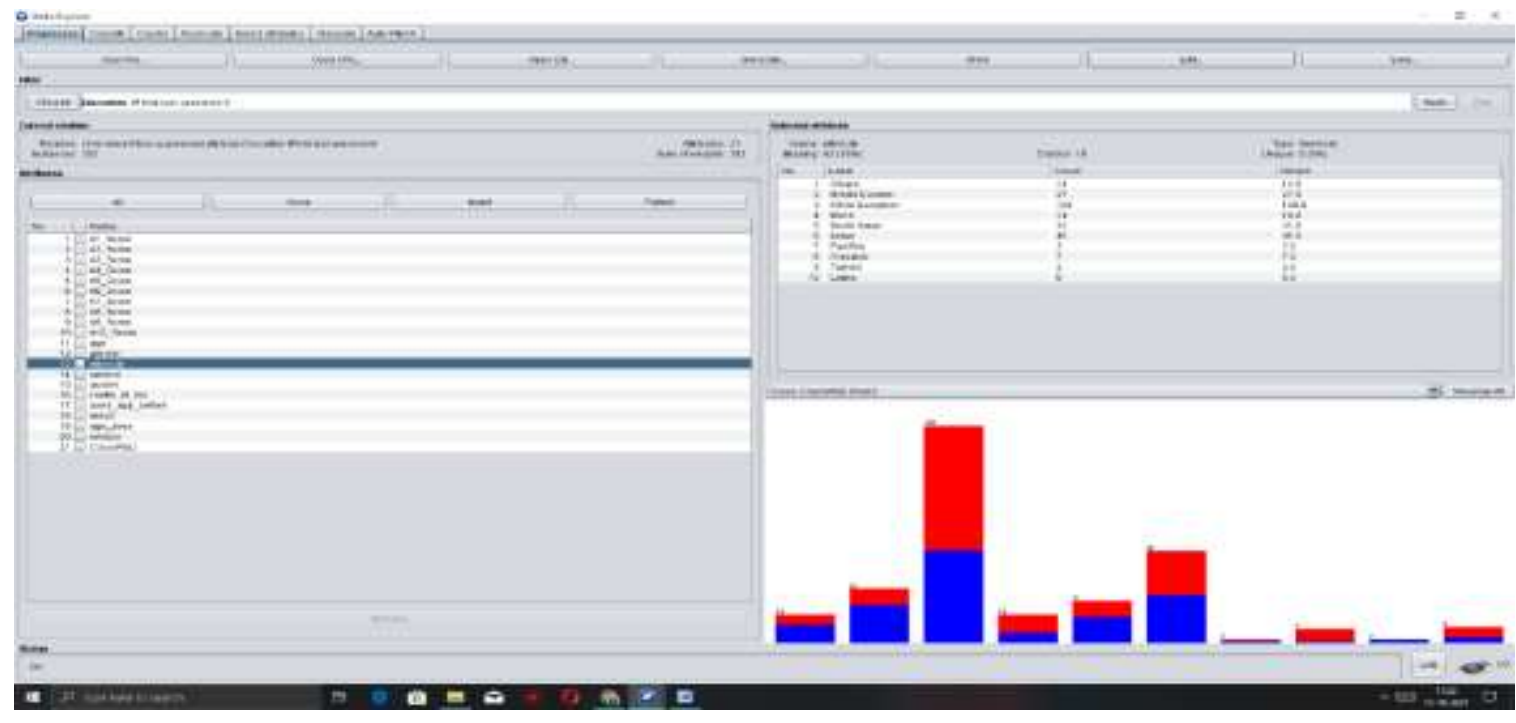

Fig. 2.1 Preprocess of the dataset using the Machine Learning tools

\subsection{NAME $=$ Weka.classifiers.rules.ZeroR}

SYNOPSIS Class for building and using a 0-R classifier. Predicts the mean (for a numeric class) or the mode (for a nominal class).

Classifier output=== Run information $===$

Scheme: weka.classifiers.rules.ZeroR

Relation: child

Instances: 292

Attributes: 21

A1_Score, A2_Score, A3_Score, A4_Score, A5_Score, A6_Score, A7_Score, A8_Score,

A9_Score, A10_Score, age, gender, ethnicity, jaundice, austim, contry_of_res, used_app_before, result, age_desc, relation, Class/ASD.

Test mode: 10-fold cross-validation

$===$ Classifier model (full training set) $===$ ZeroR predicts class value: NO

Time taken to build model: 0 seconds

$===$ Stratified cross-validation $======$ Summary $===$

Correctly Classified Instances $\quad 151 \quad 51.7123 \%$

Incorrectly Classified Instances $141 \quad 48.2877 \%$

Kappa statistic

0

Mean absolute error

0.4994

Root mean squared error

Relative absolute error

Root relative squared error

Total Number of Instances

$$
\begin{gathered}
0.4997 \\
100 \%
\end{gathered}
$$$$
100 \%
$$

292

=== Detailed Accuracy By Class ===

TP Rate FP Rate Precision Recall F-Measure MCC ROC Area PRC Area Class

$\begin{array}{ccccccccccc}1.000 & 1.000 & 0.517 & 1.000 & 0.682 & ? & 0.494 & 0.514 & \text { NO } \\ 0.000 & 0.000 & ? & 0.000 & ? & ? & 0.494 & 0.480 & \text { YES } \\ \text { Avg. } & 0.517 & 0.517 & ? & 0.517 & ? & ? & 0.494 & 0.497\end{array}$

$\begin{array}{lllllllll}\text { Weighted Avg. } & 0.517 & 0.517 & ? & 0.517 & ? & ? & 0.494 & 0.497\end{array}$

$===$ Confusion Matrix $===$

a $\mathrm{b}<--$ classified as

$1510 \mid \mathrm{a}=\mathrm{NO}$

$1410 \mid \mathrm{b}=\mathrm{YES}$ 


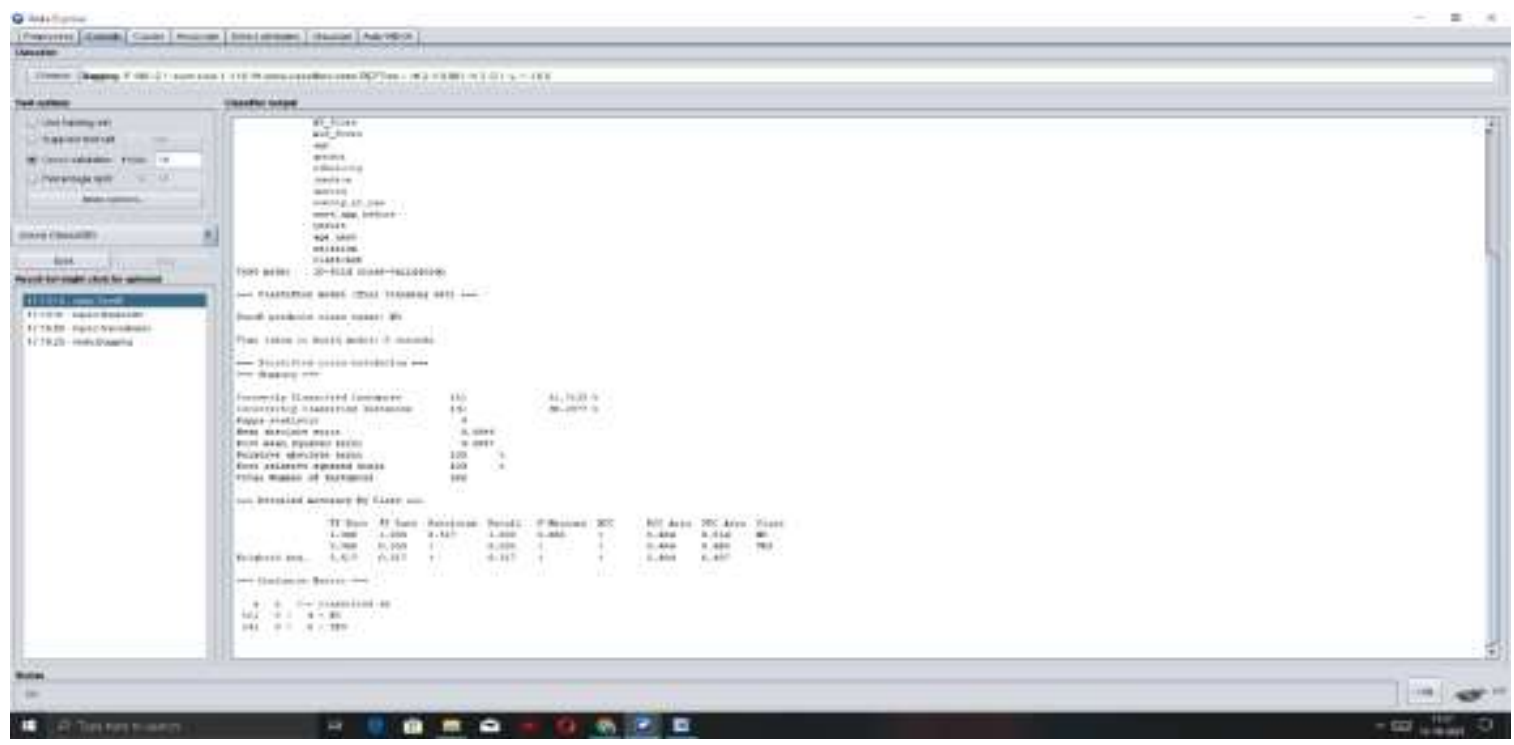

Fig. 2.2 Prediction based on ZeroR Classifier Algorithm

\subsection{NAME=Weka.classifiers.bayes.BayesNet}

\section{SYNOPSIS}

Bayes Network learning using various search algorithms and quality measures.

Base class for a Bayes Network classifier. Provides datastructures (network structure, conditional probability distributions, etc.) and facilities common to Bayes Network learning algorithms like K2 and B.

Classifier Output=== Run information $===$

Scheme: $\quad$ weka.classifiers.bayes.BayesNet -D -Q weka.classifiers.bayes.net.search.local.K2 -- -P 1 -S BAYES -E weka.classifiers.bayes.net.estimate.SimpleEstimator -- -A 0.5

Relation: child

Instances: 292

Attributes: 21

A1_Score, A2_Score, A3_Score, A4_Score, A5_Score, A6_Score, A7_Score, A8_Score,

A9_Score, A10_Score, age, gender, ethnicity, jaundice, austim, contry_of_res, used_app_before, result, age_desc, relation, Class/ASD.

Test mode: 10-fold cross-validation

$===$ Classifier model (full training set) $===$

Bayes Network Classifier

not using ADTree

\#attributes=21 \#classindex $=20$

Network structure (nodes followed by parents)

A1_Score(2): Class/ASD A2_Score(2): Class/ASD A3_Score(2): Class/ASD A4_Score(2): Class/ASD

A5_Score(2): Class/ASD A6_Score(2): Class/ASD A7_Score(2): Class/ASD A8_Score(2): Class/ASD

A9_Score(2): Class/ASD A10_Score(2): Class/ASD age(1): Class/ASD gender(2): Class/ASD

ethnicity(10): Class/ASD jundice(2): Class/ASD austim(2): Class/ASD contry_of_res(52):

Class/ASD used_app_before(2): Class/ASD result(2): Class/ASD age_desc(1): Class/ASD

relation(5): Class/ASD Class/ASD(2): LogScore Bayes: -4024.9042607655315

LogScore BDeu: -4657.8263791365225 LogScore MDL: -4557.622427621928

LogScore ENTROPY: -4106.3205003416 LogScore AIC: -4265.3205003416

Time taken to build model: 0.01 seconds

$===$ Stratified cross-validation $======$ Summary $===$

Correctly Classified Instances $\quad 292 \quad 100 \quad \%$

Incorrectly Classified Instances $00 \%$

Kappa statistic 1

Mean absolute error $\quad 0.0011$

Root mean squared error $\quad 0.0041$

Relative absolute error $\quad 0.2293 \%$

Root relative squared error $\quad 0.8265 \%$

Total Number of Instances $\quad 292$ 
$===$ Detailed Accuracy By Class ===

TP Rate FP Rate Precision Recall F-Measure MCC ROC Area PRC Area Class

$\begin{array}{ccccccccc}1.000 & 0.000 & 1.000 & 1.000 & 1.000 & 1.000 & 1.000 & 1.000 & \text { NO } \\ 1.000 & 0.000 & 1.000 & 1.000 & 1.000 & 1.000 & 1.000 & 1.000 & \text { YES } \\ \text { Avg. } & 1.000 & 0.000 & 1.000 & 1.000 & 1.000 & 1.000 & 1.000 & 1.000\end{array}$

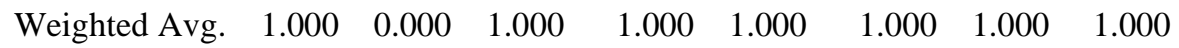

$===$ Confusion Matrix $===$

a $b \quad<--$ classified as

\begin{tabular}{ll|l}
151 & $0 \mid \mathrm{a}=\mathrm{NO}$
\end{tabular}

$0141 \mid \mathrm{b}=\mathrm{YES}$

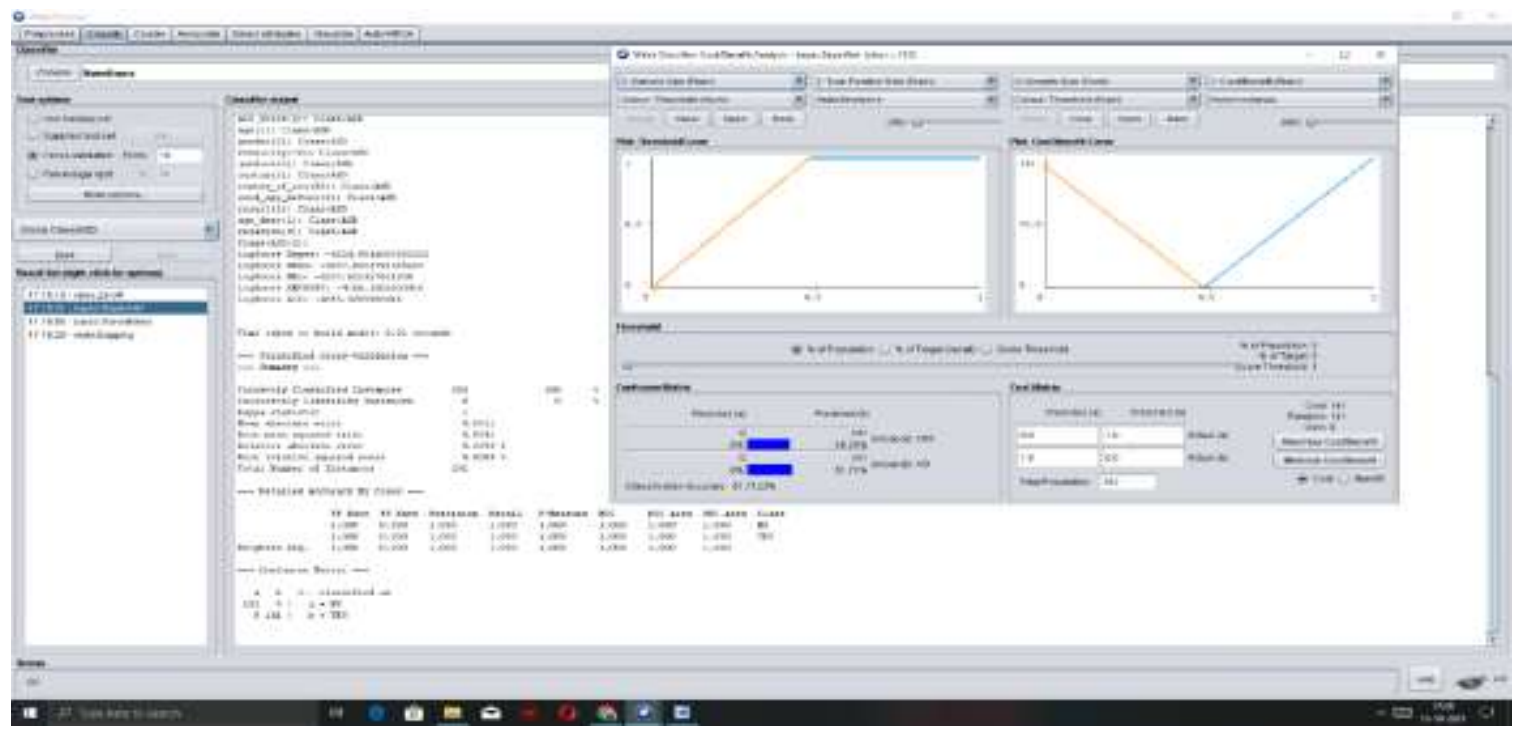

Fig. 2.3 Prediction based on BayesNetwork Algorithm with Threshold Curve

\subsection{NAME=Weka.classifiers.bayes.NaiveBayes}

\section{SYNOPSIS}

Class for a Naive Bayes classifier using estimator classes. Numeric estimator precision values are chosen based on analysis of the training data. For this reason, the classifier is not an UpdateableClassifier (which in typical usage are initialized with zero training instances) -- if you need the UpdateableClassifier functionality, use the NaiveBayesUpdateable classifier. The NaiveBayesUpdateable classifier will use a default precision of 0.1 for numeric attributes when buildClassifier is called with zero training instances.

Classifier Output-

$===$ Run information $===$

Scheme: weka.classifiers.bayes.NaiveBayes

Relation: child

Instances: 292

Attributes: 21

A1_Score, A2_Score, A3_Score, A4_Score, A5_Score, A6_Score, A7_Score, A8_Score,

A9_Score, A10_Score, age, gender, ethnicity, jaundice, austim, contry_of_res, used_app_before, result, age_desc, relation, Class/ASD.

Test mode: 10-fold cross-validation

$===$ Classifier model (full training set) $===$

Naive Bayes Classifier

Class

Attribute NO YES

(0.52) (0.48)

\begin{tabular}{|c|c|}
\hline \multicolumn{2}{|l|}{ A1_Score } \\
\hline 0 & $84.0 \quad 25.0$ \\
\hline 1 & $69.0 \quad 118.0$ \\
\hline [total] & 153.0143 .0 \\
\hline A2_Score & \\
\hline 0 & $88.0 \quad 50.0$ \\
\hline
\end{tabular}




\begin{tabular}{|c|c|}
\hline $\begin{array}{l}1 \\
\text { [total] }\end{array}$ & $\begin{array}{cc}65.0 & 93.0 \\
153.0 & 143.0\end{array}$ \\
\hline \multicolumn{2}{|l|}{ A3_Score } \\
\hline 0 & $65.0 \quad 12.0$ \\
\hline 1 & $88.0 \quad 131.0$ \\
\hline [total] & 153.0143 .0 \\
\hline \multicolumn{2}{|l|}{ A4_Score } \\
\hline 0 & $110.0 \quad 23.0$ \\
\hline 1 & $43.0 \quad 120.0$ \\
\hline [total] & $153.0 \quad 143.0$ \\
\hline \multicolumn{2}{|l|}{ A5_Score } \\
\hline $0^{-}$ & $64.0 \quad 13.0$ \\
\hline 1 & $89.0 \quad 130.0$ \\
\hline [total] & $153.0 \quad 143.0$ \\
\hline \multicolumn{2}{|l|}{ A6_Score } \\
\hline 0 & $72.0 \quad 14.0$ \\
\hline 1 & 81.0129 .0 \\
\hline [total] & $153.0 \quad 143.0$ \\
\hline \multicolumn{2}{|l|}{ A7_Score } \\
\hline $0^{-}$ & $80.0 \quad 37.0$ \\
\hline 1 & 73.0106 .0 \\
\hline [total] & $153.0 \quad 143.0$ \\
\hline \multicolumn{2}{|l|}{ A8_Score } \\
\hline 0 & $109.0 \quad 40.0$ \\
\hline 1 & $44.0 \quad 103.0$ \\
\hline [total] & $153.0 \quad 143.0$ \\
\hline \multicolumn{2}{|l|}{ A9_Score } \\
\hline 0 & $113.0 \quad 37.0$ \\
\hline 1 & $40.0 \quad 106.0$ \\
\hline [total] & 153.0143 .0 \\
\hline \multicolumn{2}{|l|}{ A10_Score } \\
\hline 0 & $71.0 \quad 11.0$ \\
\hline 1 & $82.0 \quad 132.0$ \\
\hline [total] & 153.0143 .0 \\
\hline \multicolumn{2}{|l|}{ age } \\
\hline mean & 6.18126 .5396 \\
\hline std. dev. & 2.22572 .4853 \\
\hline weight sum & $149 \quad 139$ \\
\hline $\begin{array}{l}\text { precision } \\
\text { gender }\end{array}$ & 11 \\
\hline $\mathrm{m}$ & 106.0104 .0 \\
\hline$f$ & $47.0 \quad 39.0$ \\
\hline [total] & $153.0 \quad 143.0$ \\
\hline \multicolumn{2}{|l|}{ ethnicity } \\
\hline Others & $10.0 \quad 6.0$ \\
\hline Middle Eastern & $20.0 \quad 9.0$ \\
\hline White-European & $47.0 \quad 63.0$ \\
\hline Black & $6.0 \quad 10.0$ \\
\hline South Asian & $14.0 \quad 9.0$ \\
\hline Asian & $25.0 \quad 23.0$ \\
\hline Pasifika & $2.0 \quad 2.0$ \\
\hline Hispanic & $2.0 \quad 7.0$ \\
\hline Turkish & $3.0 \quad 1.0$ \\
\hline Latino & $4.0 \quad 6.0$ \\
\hline [total] & 133.0136 .0 \\
\hline \multicolumn{2}{|l|}{ jundice } \\
\hline no & 109.0105 .0 \\
\hline yes & $44.0 \quad 38.0$ \\
\hline [total] & $153.0 \quad 143.0$ \\
\hline
\end{tabular}

DOI: $10.17148 /$ IJARCCE.2021.101102 
austim

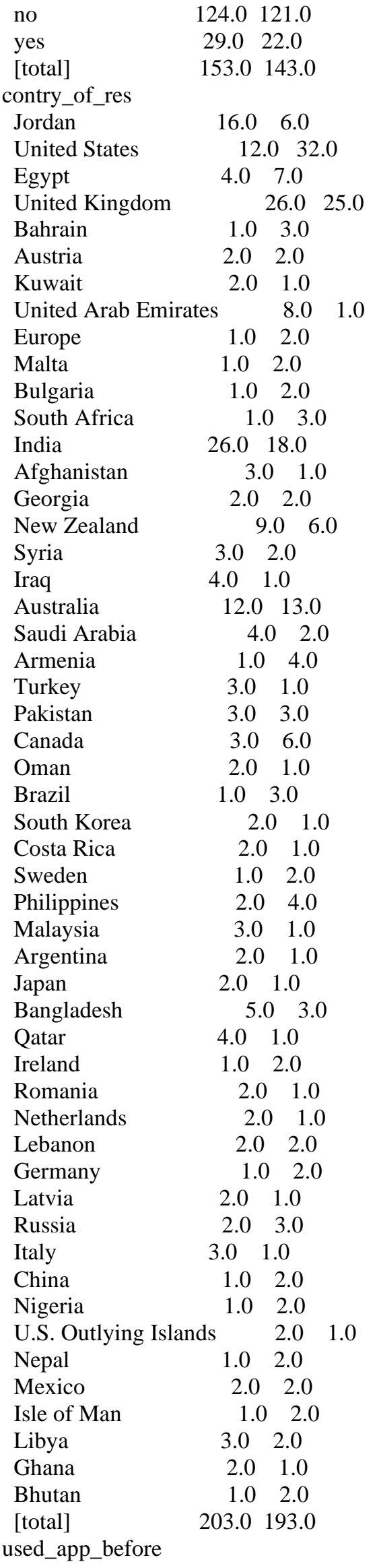




\begin{tabular}{|c|c|}
\hline no & 145.0138 .0 \\
\hline yes & $8.0 \quad 5.0$ \\
\hline [total] & $153.0 \quad 143.0$ \\
\hline \multicolumn{2}{|l|}{ result } \\
\hline mean & 4.39748 .2128 \\
\hline std. dev. & 1.41931 .043 \\
\hline weight sum & $151 \quad 14$ \\
\hline precision & 11 \\
\hline \multicolumn{2}{|l|}{ age_desc } \\
\hline 4-11 years & 152.0142 \\
\hline [total] & $152.0 \quad 142.0$ \\
\hline \multicolumn{2}{|l|}{ relation } \\
\hline Parent & 103.0113 .0 \\
\hline Self & $4.0 \quad 2.0$ \\
\hline Relative & $13.0 \quad 6.0$ \\
\hline
\end{tabular}

Health care professional $\quad 7.0 \quad 8.0$

$$
\begin{array}{lcl}
\text { self } & 1.0 & 2.0 \\
\text { [total] } & 128.0 & 131.0
\end{array}
$$

Time taken to build model: 0 seconds

$===$ Stratified cross-validation $===$

$===$ Summary $===$

Correctly Classified Instances 28

Incorrectly Classified Instances 3

Kappa statistic

0.9794

Mean absolute error

0.0444

Root mean squared error

Relative absolute error

Root relative squared error

Total Number of Instances

=== Detailed Accuracy By Class ===

TP Rate FP Rate Precision Recall F-Measure MCC ROC Area PRC Area Class

$\begin{array}{lllllllll}0.987 & 0.007 & 0.993 & 0.987 & 0.990 & 0.979 & 1.000 & 1.000 & \text { NO } \\ 0.993 & 0.013 & 0.986 & 0.993 & 0.989 & 0.979 & 1.000 & 1.000 & \text { YES }\end{array}$

$\begin{array}{lllllllll}\text { Weighted Avg. } & 0.990 & 0.010 & 0.990 & 0.990 & 0.990 & 0.979 & 1.000 & 1.000\end{array}$

$===$ Confusion Matrix $===$

a $b$ <- classified as

1492 | $\mathrm{a}=\mathrm{NO}$

$1140 \mid \mathrm{b}=\mathrm{YES}$

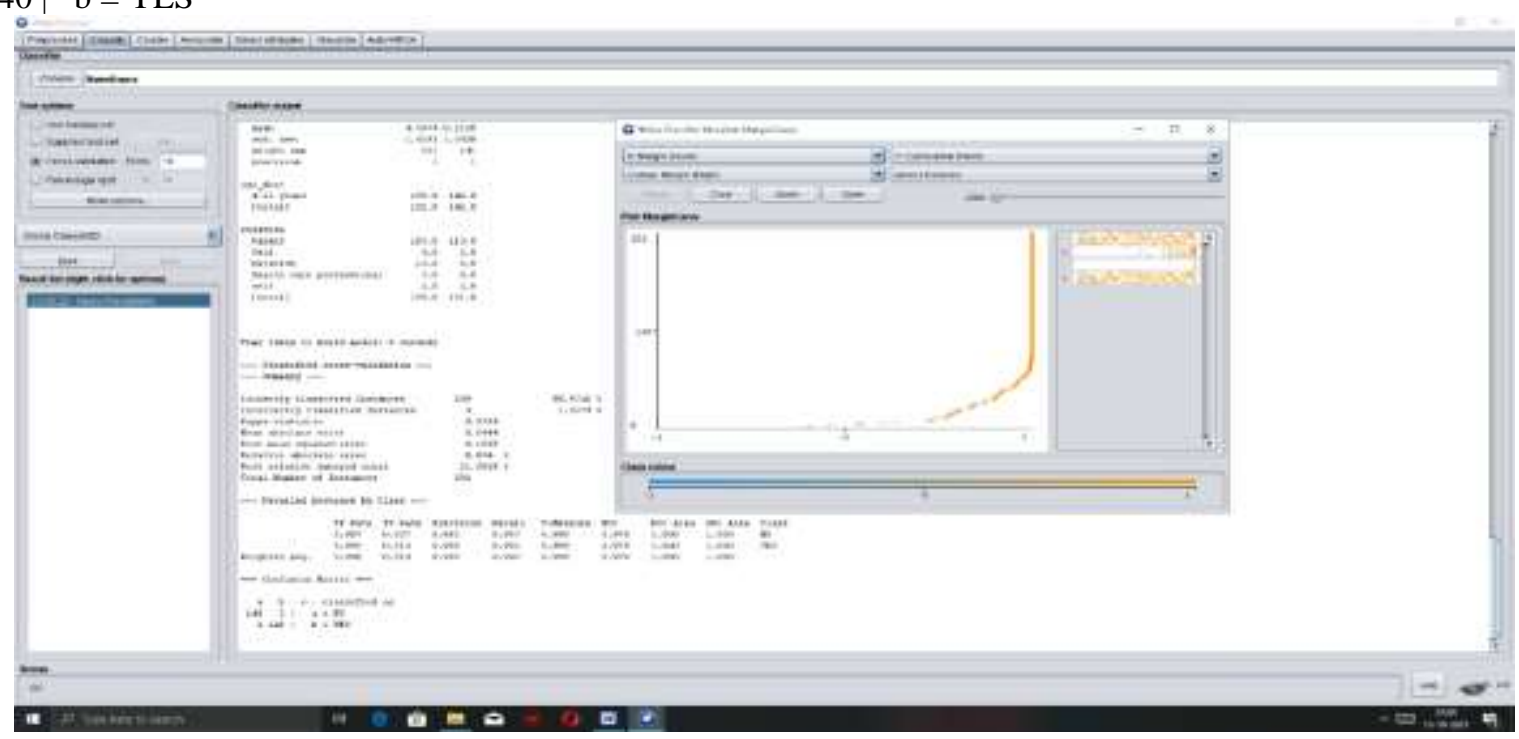

Fig. 2.4 Prediction Based on NaiveBayes Algorithm with Margin Curve 


\section{International Journal of Advanced Research in Computer and Communication Engineering}

Vol. 10, Issue 11, November 2021

DOI: $10.17148 /$ IJARCCE.2021.101102

\section{DISCUSSION}

The psychosocial disorder with the cardinal effect of pandemic and children in adolescents is a disruption of their lives[7]. Disruption leads to a loss of predictability, reliability etc. Toddlers respond with increased dependency. School children show evidence of trauma with play and talk about trauma and hostility to family. Adolescents may also withdraw and have decreased interest and experience fatigue, hypertension, hostility and loss of objectivity[8][9]. It is impossible to separate the effects of disasters on children and their families and the two should be considered as a unit. In this paper we have used 4 different machine learning algorithms to analyse and predict the accuracy from the given dataset. As results from experiments and observation shows that, the Naïve bayes classifier algorithm is an efficient algorithm which gives more accurate results of the correctly classified instances of $98.9726 \%$.

\section{CONCLUSION}

No doubt, educational technology can make education and learning effective and interesting, but Children need to be viewed as an integral part of the population, not a "special case" to be dealt with separately. In this paper, we have shown some real pictures on the Psychosocial Disorder in children and changes in there behavior after the pandemic period in educational perspective. A clear analysis by using machine learning approach on this study shown that, the pandemic has affect more on the psychosocial level on children. Machine Learning will be an excellent tool to predict, analysis and observation for this kind of real issues to resolve for the effectiveness, efficient, and accurate result to be used in the near future.

\section{REFERENCES}

[1] Popa, S. Reflections on COVID-19 and the future of education and learning. Prospects 49, 1-6 (2020). https://doi.org/10.1007/s11125-02009511-Z

[2] Dalton, L., Rapa, E., and Stein, A. (2020). Protecting the psychological health of children through effective communication about COVID19. Lancet 4, 346-347. doi: 10.1016/S2352-4642(20)30097-3

[3] Ui DS, I Azhar E, Madani TA, Ntoumi F, Kock R, Dar O, et al. The continuing 2019-nCoV epidemic threat of novel coronaviruses to global health-The latest 2019 novel coronavirus outbreak in Wuhan, China. International Journal of Infectious Diseases. 2020;91:264-6. pmid:31953166

[4] Liu, J. J., Bao, Y., Huang, X., Shi, J., and Lu, L. (2020). Mental health considerations for children quarantined because of COVID19. Lancet 4, 347-349. doi: 10.1016/S2352-4642(20)30096-1

[5] Onyema EM, Eucheria NC, Obafemi FA, Sen S, Atonye FG, Sharma A, et al. Impact of Coronavirus pandemic on education. Journal of Education and Practice. 2020;11(13):108-21.

[6] Adnan M, Anwar K. Online Learning amid the COVID-19 Pandemic: Students' Perspectives. Online Submission. 2020;2(1):45-51.

[7] Cao W, Fang Z, Hou G, Han M, Xu X, Dong J, et al. The psychological impact of the COVID-19 epidemic on college students in China. Psychiatry research. 2020;287:112934. pmid:32229390; 32229390.

[8] Sahu P. Closure of Universities Due to Coronavirus Disease 2019 (COVID-19): Impact on Education and Mental Health of Students and Academic Staff. 2020;12(4). pmid:32377489

[9] Poh-Sun G, John S. A vision of the use of technology in medical education after the COVID-19 pandemic. MedEdPublish. 2020;9(1):49. edsdoj.9b8f5410675446488c9f35323d72c0c9.

[10] Latané B. The psychology of social impact. American Psychologist. 1981;36(4):343-56.

[11] Xia S, Liu J. A computational approach to characterizing the impact of social influence on individuals' vaccination decision making. PloS one. 2013;8(4):e60373. pmid:23585835.

[12] Cohen J. Statistical power analysis for the behavioral sciences. 2nd ed: Hillsdale, N.J.: L. Erlbaum Associates; 1988 .

[13] Andel SA, de Vreede T, Spector PE, Padmanabhan B, Singh VK, de Vreede G-J. Do social features help in video-centric online learning platforms? A social presence perspective. Computers in Human Behavior. 2020;113. S0747563220302570.

[14] Vargas, C. (2019), "Leaving no one behind: bringing equity and inclusion back into education", Resisting Neoliberalism in Education:

Local, National and Transnational Perspectives, L. Tett and M. Hamilton (eds.), Bristol, Policy Press.

[15] Cortis, D. (2020). On determining the age distribution of COVID-19 Pandemic. Front. Public Health 8:202. doi:

10.3389/fpubh.2020.00202

\section{BIOGRAPHY}

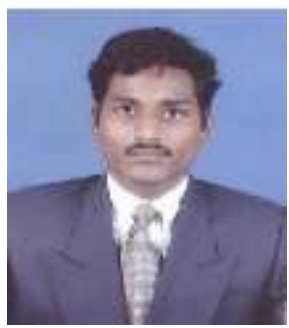

Mr. Dillip Narayan Sahu received his B.Sc. degree in physics from Sambalpur University, India, Master of Computer Application degree from Sambalpur University, India, M.Tech. degree in Computer Science from Sambalpur University, India, M-Phil degree Computer Science from MATS University, India, and continuing Ph.D. in Computer Science. His research area includes Artificial Intelligence, Machine Learning, Analysis and Design of Algorithms, Data Science, IoT. He has published many papers in National/International Conferences, Seminars, and Journals. Presently He is working as a Lecturer in the Dept. of MCA, Gangadhar Meher University, India. 
Vol. 10, Issue 11, November 2021

DOI: $10.17148 /$ IJARCCE.2021.101102

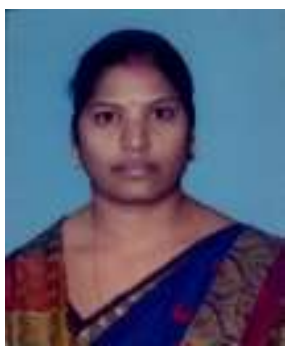

Ms. Pankajini Sahu received her M.Phil. degree in Education from MATS University, India. She has wide publications in many national, International journals and Conferences. Her research area includes Educational Psychology, Primary Education, Learner and learning, Teacher and classroom, Information and Communication Technology (ICT). Presently she is working as a Lecturer in Dept. of Education, ShreeRam College, Rampur, Sonepur, Odisha, India. 